\title{
Suppression of plasminogen activator inhibitor- 1 by RNA interference attenuates pulmonary fibrosis
}

\author{
Tadashi Senoo, ${ }^{1}$ Noboru Hattori, ${ }^{1}$ Takuya Tanimoto, ${ }^{1}$ Makoto Furonaka, ${ }^{1}$ \\ Nobuhisa Ishikawa, ${ }^{1}$ Kazunori Fujitaka, ${ }^{1}$ Yoshinori Haruta, ${ }^{1}$ Hiroshi Murai, ${ }^{1}$ \\ Akihito Yokoyama, ${ }^{2}$ Nobuoki Kohno ${ }^{1}$
}

\begin{abstract}
- Supplementary methods and figures are published online only at http://thorax.bmj.com/ content/vol65/issue4

${ }^{1}$ Department of Molecular and Internal Medicine, Graduate School of Biomedical Sciences, Hiroshima University, Hiroshima, Japan

${ }^{2}$ Department of Hematology and Respiratory Medicine, Koch University, Kochi, Japan
\end{abstract}

Correspondence to Dr Noboru Hattori, Department of Molecular and Internal Medicine, Graduate School of Biomedical, Sciences, Hiroshima University, 1-2-3 Kasumi, Minami-ku, Hiroshima 734-8551, Japan; nhattori@hiroshima-u.ac.jp

Received 18 May 2009 Accepted 24 January 2010

\section{ABSTRACT}

Background and aim There is a growing body of evidence demonstrating that plasminogen activator inhibitor-1 (PAI-1) is involved in the progression of pulmonary fibrosis. In fact, PAl-1 knockout mice are protected from bleomycin-induced pulmonary fibrosis. This study was conducted to determine whether the intrapulmonary administration of small interfering RNA (siRNA) targeting PAI-1 (PAI-1-siRNA) limits the development of bleomycin-induced pulmonary fibrosis. Methods Lung biopsies from patients with idiopathic pulmonary fibrosis (IPF) were stained for PAI-1. The distribution of siRNA in the lung, the PAl-1 level in bronchoalveolar (BAL) fluid and the extent of fibrotic changes in the lung were evaluated following the intranasal administration of PAI-1-siRNA in a mouse model of bleomycin-induced pulmonary fibrosis. The effect of PAI-1-siRNA on the epithelial to mesenchymal transition (EMT) was also evaluated using a mouse lung epithelial cell line, LA-4.

Results PAI- 1 was overexpressed in the hyperplastic type 2 pneumocytes lining the honeycomb lesions of patients with IPF. The single intranasal instillation of PAI1-siRNA resulted in the diffuse uptake of siRNA into the epithelial cells lining the dense fibrotic lesions. The repeated administration of PAI-1-siRNA initiated during either the inflammatory or the fibrotic phase into bleomycin-injured mice reduced the PAl-1 level in BAL fluid and limited the accumulation of collagen in the lungs. EMT induced by transforming growth factor $\beta$ (TGF $\beta$ ) in LA-4 cells was inhibited by transfection with PAl-1-siRNA.

Conclusions The direct suppression of PAI-1 in the lung by the intrapulmonary administration of PAI-1-siRNA attenuated the development and progression of pulmonary fibrosis. The inhibition of EMT may be, at least in part, involved in this effect.

\section{INTRODUCTION}

Fibrin accumulation is a common feature in a variety of acute and chronic pulmonary diseases. Damage to the alveolar walls causes extravasation of plasma proteins into the interstitium and alveolar spaces where increased levels of tissue factor lead to the accumulation of fibrin and other matrix components. ${ }^{12}$ The fate of this provisional matrix is an important determinant of whether the alveolar structures are successfully repaired or replaced by collagenous scars. The urokinase-type plasminogen activator (uPA) is produced by pulmonary epithelial cells ${ }^{3}{ }^{4}$ and alveolar macrophages in a normal lung, ${ }^{5}$ thereby generating a net fibrinolytic environment in the alveolar space. However, fibrin deposits persist in patients with fibrotic lung diseases because the normal fibrinolytic activity is suppressed by an increased expression of plasminogen activator inhibitor-1 (PAI-1). ${ }^{6-8}$

Manipulation of the PAI-1 levels in animal models of lung injury influences the degree of fibrosis that occurs. Mice with a targeted deletion of the PAI-1 gene (PAI-1 ${ }^{-/-}$mice) develop less fibrosis and survive longer following bleomycin injury in comparison with wild-type mice, while transgenic mice that overexpress PAI-1 developed more severe bleomycin-induced pulmonary fibrosis. ${ }^{9}$ These observations suggest a direct correlation between the expression level of PAI-1 and the extent of collagen accumulation that follows lung injury, and, therefore, PAI-1 can be recognised as a key molecule involved in the development of pulmonary fibrosis. Furthermore, these observations suggest that the suppression of PAI-1 in the injured lung can therefore be a therapeutic strategy to limit the development of pulmonary fibrosis. In the current study, small interfering RNA (siRNA) directed against PAI-1 (PAI-1-siRNA) was delivered into the lungs of mice through an intranasal route. This strategy was selected because previous studies have shown the intranasal administration of siRNA to be effective for the lung-specific knockdown of gene expression, and it can be easily and safely repeated. ${ }^{10} 11$

The present study investigated which regions in the lung received the PAI-1-siRNA and whether the intranasal administration of PAI-1-siRNA could reduce the PAI-1 level in the lung using a murine model of bleomycin-induced pulmonary fibrosis. Furthermore, these mice were evaluated to determine whether the repeated instillation of PAI-1siRNA could limit the development of pulmonary fibrosis. In addition, the effect of PAI-1-siRNA on epithelial to mesenchymal transition (EMT) induced by transforming growth factor $\beta$ (TGF $\beta$ ) was also evaluated using a mouse lung epithelial cell line.

\section{METHODS \\ SiRNA design}

The siRNAs were designed using the TaKaRa online algorithm (TaKaRa Bio, Tokyo, Japan). See online supplement for details.

\section{Bleomycin exposure and the administration of SiRNA}

Wild-type C57BL/6 mice were purchased from CLEA Japan (Tokyo, Japan). The mice were 
anaesthetised with intraperitoneal pentobarbital, and the trachea was exposed through a cervical incision. Bleomycin (1.5 mg/kg body weight, Nippon Kayaku, Tokyo, Japan) was dissolved in phosphate-buffered saline (PBS) and then instilled intratracheally with a 27-gauge needle. A higher dose of bleomycin ( $2.5 \mathrm{mg} / \mathrm{kg}$ body weight) was used to assess survival. PAI1-siRNA and non-specific siRNA(NS-siRNA) were dissolved in RNase-free water and then administered intranasally $(2 \mu \mathrm{M}$ in $50 \mu \mathrm{l})$ after anaesthetising the mice with pentobarbital.

\section{Immunohistochemistry for PAI-1}

Immunohistochemical analysis of PAI-1 was performed as described in the online supplement.

\section{Bronchoalveolar lavage (BAL)}

BAL was performed as described in the online supplement.

\section{Measurement of PAI-1 level and uPA activity in BAL fluid}

The levels of PAI-1 and the activity of functionally active uPA in BAL fluid were measured using a total murine PAI-1 antigen assay kit (Innovative Research, Novi, Michigan, USA) and a mouse uPA activity assay kit (Innovative Research), respectively, following the manufacturer's instructions.

\section{Confocal laser scanning microscopy}

Rhodamine-labelled PAI-1-siRNA was synthesised by TaKaRa Bio (Tokyo, Japan) and instilled intranasally into mice. Twentyfour hours after siRNA instillation, frozen lung sections and cells collected in BAL fluid were scanned at an excitation wavelength of $488 \mathrm{~nm}$ for rhodamine using an LSM5 Pascal confocal imaging system (Carl Zeiss, Jena, Germany) with an inverted microscope. The means of eight scans were obtained at a resolution of $1024 \times 1024$ pixels. Confocal images were stored as digital files and viewed using Photoshop (Adobe, Mountain View, California, USA).

\section{Lung collagen measurement}

The degree of pulmonary fibrosis was determined by measuring total soluble lung collagen using the Sircol Collagen Assay kit (Biocolor, Belfast, UK) as described previously. ${ }^{12} 13$

\section{Histology}

After BAL and lung perfusion, the lungs of mice were fixed by inflation with a buffered $10 \%$ formalin solution. The lung tissue specimens were embedded in paraffin, and then the sections were stained with $\mathrm{H} \& \mathrm{E}$ for examination by light microscopy.

\section{Assessment of cell proliferation}

Cell proliferation following siRNA transfection was analysed using a mouse lung epithelial cell line, LA-4. See the online supplement for details.

\section{Assessment of EMT and apoptosis}

LA-4 cells grown in 8-well chamber slides were transfected with PAI-1-siRNA (100 nM) or NS-siRNA (100 nM) using Trans ITTKO. TGF $\beta 1$ at $5 \mathrm{ng} / \mathrm{ml}$ was added to the culture $48 \mathrm{~h}$ after the transfection, and then the cells were incubated for another $48 \mathrm{~h}$. The cells were fixed in $4 \%$ paraformaldehyde and treated with $3 \% \mathrm{H}_{2} \mathrm{O}_{2}$ in methanol for $15 \mathrm{~min}$. Following the blocking step, the cells were incubated with a rabbit polyclonal antibody against $\boldsymbol{\alpha}$-smooth muscle actin ((SMA) ab5694; Abcam, Cambridge, UK) labelled with Zenon Alexa Fluor 488 and a rabbit polyclonal antibody against E-cadherin (sc-7870; Santa Cruz Biotechnology, Santa Cruz, California, USA) labelled with
Zenon Alexa Fluor 594 for $1 \mathrm{~h}$. Thereafter, the cells were mounted and examined by the BZ-9000 fluorescence imaging system (Keyence, Osaka, Japan). The degree of EMT was quantified by counting the number of $\alpha$-SMA-positive cells in seven low-power fields per sample. Apoptosis of LA-4 cells was evaluated using the ApopTag Peroxidase In Situ Apoptosis Detection Kit (Millipore, Billerica, Massachusetts, USA) following the manufacturer's instruction. The degree of apoptosis was quantified by counting the number of TUNEL (terminal deoxynucleotidyl transferase-mediated dUTP nick end labelling)-positive cells in seven low-power fields per sample.

\section{Statistical analysis}

The results are expressed as the means \pm SEM. The statistical significances of differences were analysed by the Mann-Whitney $U$ test or Student $t$ test. The proliferation of LA-4 cells was statistically analysed using repeated measures analysis of variance (ANOVA). A p value of $<0.05$ was considered to be statistically significant.

\section{RESULTS}

\section{Localisation of PAI-1 in lungs of patients with IPF}

Although one previous study conducted an immunohistochemical analysis of PAI-1 in the lungs of patients with IPF, the localisation of PAI-1 in such lungs has not been conclusively demonstrated. PAI-1 was immunohistochemically determined in the lungs of three patients with the final histopathological diagnosis of IPF. PAI-1 was diffusely expressed in the lungs of patients with IPF (Supplementary figure 1C,D). PAI-1 was strongly expressed in the areas of columnar epithelial cells lining the honeycomb lung, the hyperplastic type 2 pneumocytes and the alveolar macrophages. No significant staining was detected in the IPF lung sections that reacted with normal mouse immunoglobulin G (Supplementary figure 1B). PAI-1 expression was also observed in alveolar macrophages in the normal lung control (Supplementary figure $1 \mathrm{~A}$ ).

\section{Localisation of PAI-1-siRNA in lungs of normal mice and bleomycin-injured mice following a single intranasal administration}

Rhodamine-labelled PAI-1-siRNA was intranasally instilled into normal mice and mice intratracheally administered with bleomycin 14 days earlier, and the localisation of fluorescence in the lung was examined by confocal microscopy. The fluorescence intensity in the lungs of normal mice was observed in the bronchial epithelium and alveolar epithelium (figure 1A). The highest fluorescence intensity in the lungs of mice with bleomycin-induced pulmonary fibrosis was observed in the area associated with the bronchial epithelium, and slightly less intense fluorescence emanated from the cells covering the dense fibrotic lesion (figure 1B). BAL was performed on bleomycininjured mice intranasally instilled with rhodamine-labelled PAI1-siRNA $24 \mathrm{~h}$ earlier, and the fluorescence in the cells collected in the BAL fluid was examined by confocal microscopy. As shown in figure $1 \mathrm{C}$, the fluorescence was detected in the macrophagelike cells but not in the lymphocyte-like cells.

Single intranasal administration of PAI-1-siRNA reduced the PAI1 level in BAL fluid from mice with bleomycin-induced lung injury The PAI-1 levels in BAL fluids were measured after the intranasal instillation of PAI-1-siRNA into mice with bleomycin-induced lung injury to determine whether a single intranasal administration of PAI-1-siRNA is effective in reducing PAI-1 expression in the lung. A single intranasal administration of PAI-1-siRNA 
A
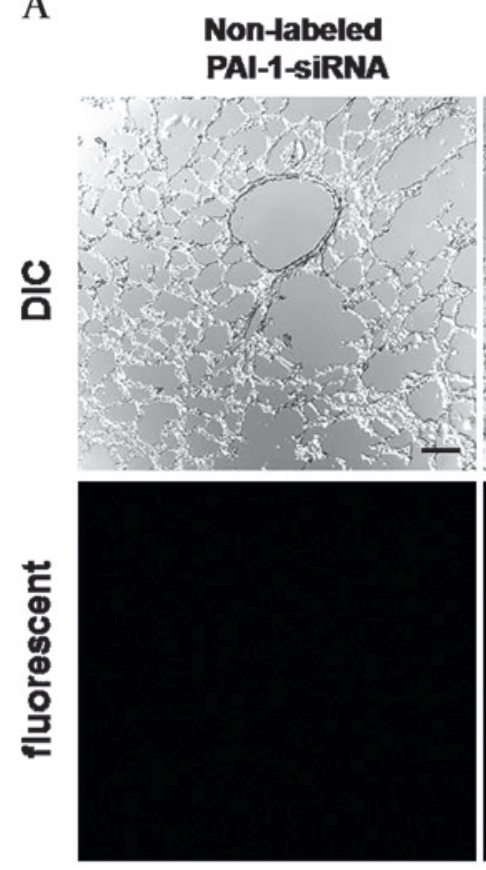

C

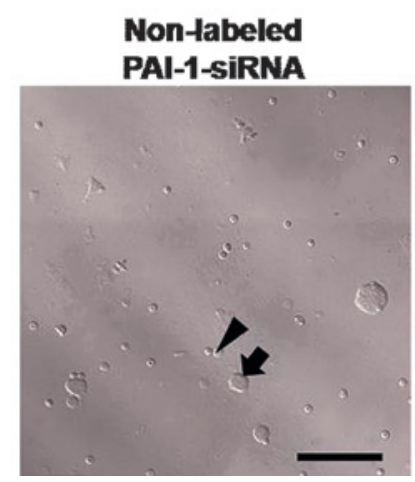

Rhodamine-labeled PAI-1-siRNA
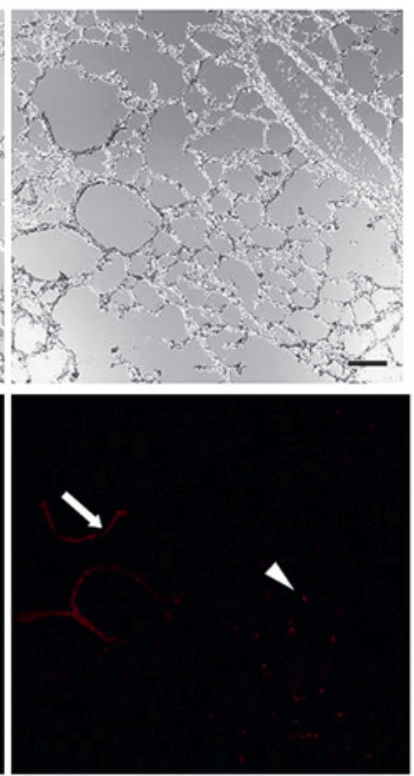

Rhodamine-labeled PAI-1-SiRNA

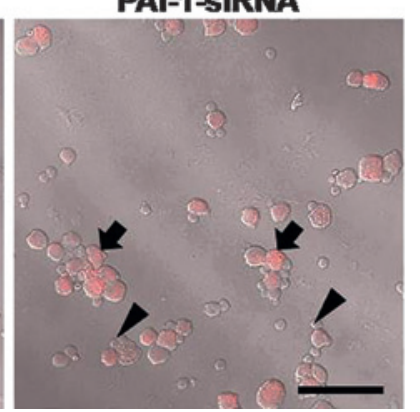

B

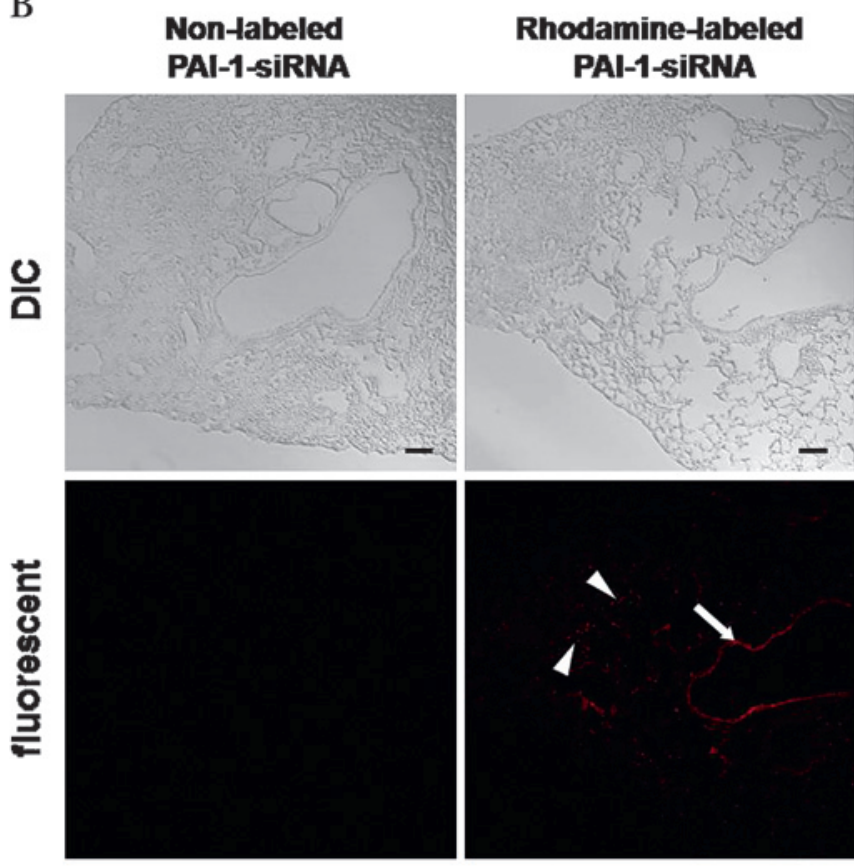

Figure 1 Localisation of plasminogen activator inhibitor-1 small interfering RNA (PAI-1-siRNA) following a single intranasal administration into normal mice or mice with bleomycin-induced pulmonary fibrosis. Rhodamine-labelled PAI-1-siRNA and non-labelled PAI-1-siRNA were intranasally instilled into normal mice or mice administered bleomycin 14 days previously. Twenty-four hours after the siRNA instillation, frozen lung sections were prepared and observed by confocal fluorescence microscopy. The smears of the cells harvested in bronchoalveolar lavage (BAL) fluid were prepared and also observed by confocal fluorescence microscopy. Representative differential interference contrast (DIC) images and confocal fluorescence microscopy images of lung sections and cell smears were taken. (A) The lung sections of the normal mice. Fluorescence emanated from bronchial epithelium (arrows) and alveolar epithelium (arrowheads). Scale bars, $100 \mu \mathrm{m}$. (B) The lung sections of the mice with bleomycin-induced pulmonary fibrosis. Fluorescence emanated from the bronchial epithelium (arrows) and the cells lining the dense fibrotic lesion (arrowheads). Scale bars, $100 \mu \mathrm{m}$. (C) The smears of the cells harvested in BAL fluid from the mice with bleomycin-induced pulmonary fibrosis. DIC and confocal fluorescent images were overlaid. The arrows and arrowheads indicate macrophage-like cells and lymphocyte-like cells, respectively. Scale bars, $50 \mu \mathrm{m}$.

reduced the PAI-1 concentration in BAL fluids by $>50 \%$ in comparison with that of the mice that received a single intranasal administration of NS-siRNA $(p<0.05$; Supplementary figure 2).

\section{Repeated administration of PAI-1-siRNA into bleomycin-injured} mice initiated during the inflammatory phase reduced fibrosis and improved survival

Following the successful reduction of the PAI-1 level in the BAL fluid in mice with bleomycin-induced lung injury after a single intranasal administration of PAI-1-siRNA, experiments were conducted to determine the effect of repeated intranasal instillation of PAI-1-siRNA on bleomycin-induced lung injury and fibrosis. PAI-1-siRNA and NS-siRNA were intranasally instilled into mice $1,4,8$, and 11 days after the intratracheal adminis- tration of bleomycin. First, the inflammatory cells recovered in the BAL fluids collected on day 7 were analysed, and measurement of the PAI-1 levels in BAL fluids, collagen contents in the lungs and a histological analysis of the lungs were performed on day 14 . The recovery rate of BAL fluid typically exceeded $90 \%$ and did not differ between the experimental groups. The number of total inflammatory cells recovered in the BAL fluids on day 7 was significantly elevated by the intratracheal administration of bleomycin; however, no significant differences were observed in the total cell counts between the mice intranasally administered PAI-1-siRNA and NS-siRNA (Supplementary figure $3 \mathrm{~A}$ ). The differential counts of the inflammatory cells in BAL fluids also showed that the fractions of macrophages, lymphocytes and neutrophils did not differ between the mice administered PAI-1-siRNA and NS-siRNA (Supplementary 
figure $3 \mathrm{~B}-\mathrm{D})$. The repeated intranasal instillation of PAI-1siRNA was found to reduce the PAI-1 level (figure 2A) and increase uPA activity (figure $2 \mathrm{~B}$ ) in BAL fluids. Immunohistochemical analysis of PAI-1 also demonstrated that the expression level of PAI-1 was lower in the bleomycin-injured mice treated with PAI-1-siRNA in comparison with that in NS-siRNA-treated mice (figure 2C). In addition, the repeated intranasal instillation of PAI-1-siRNA was able effectively to limit the accumulation of collagen in the lung in comparison with NS-siRNA in mice with bleomycin-induced lung injury (figure 2D). A histological examination also revealed that the bleomycin-induced fibrosis improved in the mice repeatedly administered PAI-1-siRNA (figure 2E). Furthermore, the repeated administration of PAI-1siRNA significantly improved the survival rate in comparison with NS-siRNA in mice with bleomycin-induced lung injury (Supplementary figure 4).

\section{Repeated administration of PAI-1-siRNA to bleomycin-injured mice initiated during the fibrotic phase reduced fibrosis}

The repeated administration of PAI-1-siRNA was initiated during the fibrotic phase of bleomycin-induced lung injury to determine whether the repeated intranasal administration of PAI-1-siRNA has an antifibrotic effect. PAI-1-siRNA and NSsiRNA were intranasally instilled 8, 11, 14, 17 and 20 days after the intratracheal administration of bleomycin, and the collagen content in the lungs obtained on day 21 was measured. The repeated intranasal instillation of PAI-1-siRNA during the fibrotic phase of bleomycin-induced lung injury was found to reduce the PAI-1 level (figure 3A), increase the uPA activity (figure $3 \mathrm{~B}$ ) in BAL fluids and limit the accumulation of collagen in the lung in comparison with NS-siRNA (figure 3C).

\section{Transfection with PAI-1-siRNA did not affect the cell proliferation rate in a mouse lung epithelial cell line}

Cell proliferation was compared between LA-4 cells transfected with PAI-1-siRNA and NS-siRNA to assess whether cell proliferation is affected by the introduction of PAI-1-siRNA into lung epithelial cells. No significant differences in cell proliferation rates were found at 24 and $48 \mathrm{~h}$ after the transfection with siRNA (data not shown).
A

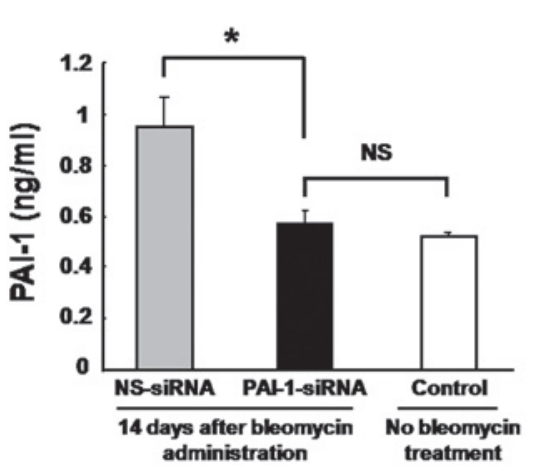

D

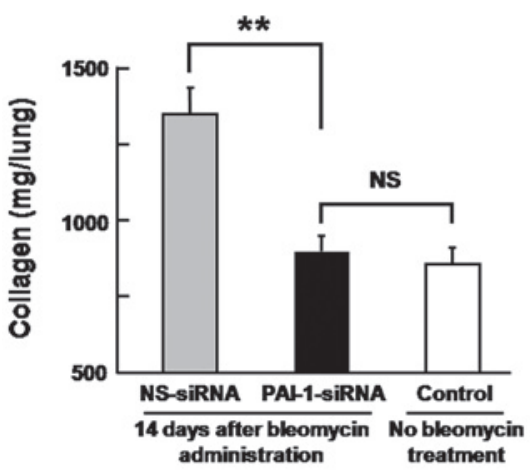

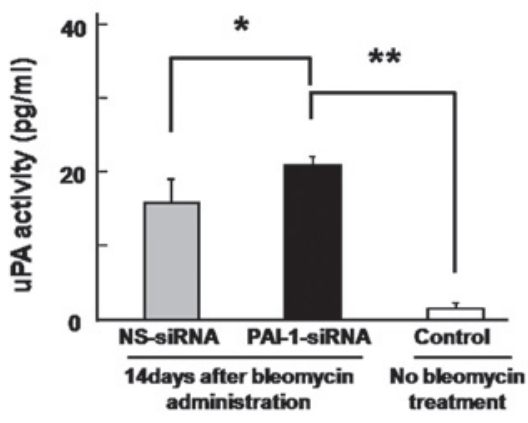

$\mathrm{E}$

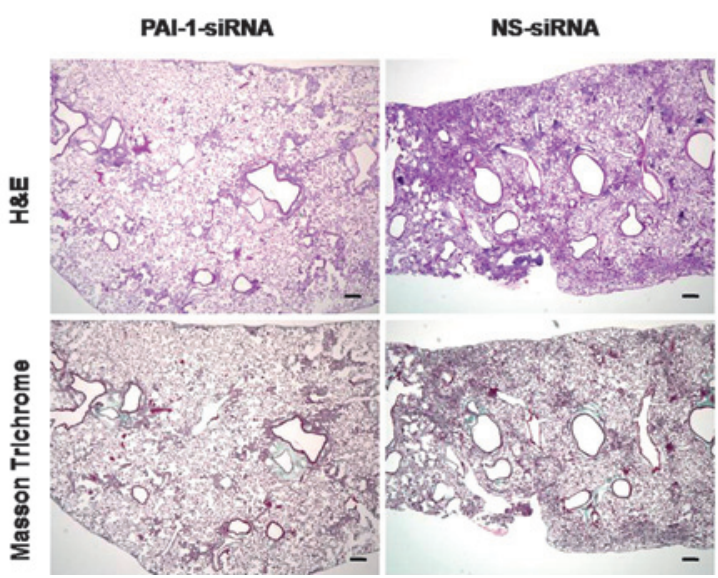

C

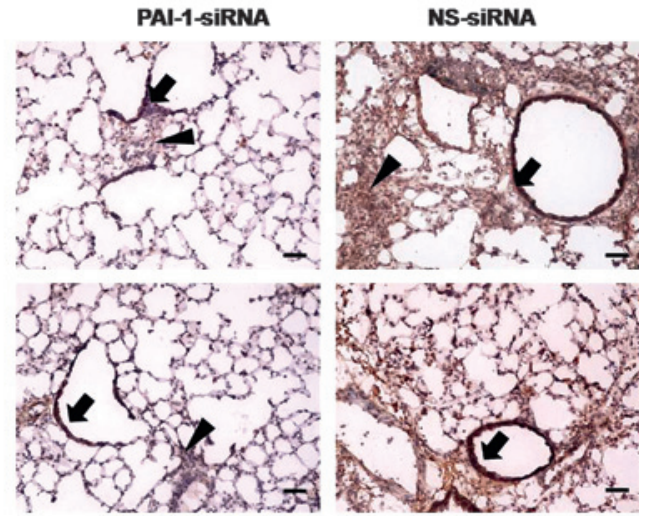

Figure 2 Effect of repeated intranasal administration of plasminogen activator inhibitor-1 small interfering RNA (PAI-1-siRNA) on the PAI-1 level, urokinase-type plasminogen activator (uPA) activity and collagen accumulation in the lungs. PAl-1-siRNA or non-specific siRNA (NS-siRNA) was intranasally instilled into the lungs of mice 1, 4, 8 and 11 days after bleomycin administration (1.5 mg/kg body weight). (A) Fourteen days after bleomycin administration, bronchoalveolar lavage (BAL) fluid was collected and the PAI-1 level in the supernatant was measured using an ELISA kit for murine PAl-1. Data are shown as the mean \pm SEM for eight mice per group. ${ }^{*} p<0.05$ vs NS-siRNA treatment. (B) Fourteen days after bleomycin administration, BAL fluid was collected and the UPA activity in the supernatant, which indicates the level of uPA not bound to PAls, was measured using a murine uPA activity assay kit. Data are shown as the mean \pm SEM for eight mice per group. ${ }^{*} p<0.05$ vs NS-siRNA treatment; ${ }^{* *} p<0.01$ vs control mice. (C) PAI-1 expression in the lungs was immunohistochemically analysed. The arrows and arrowheads indicate the bronchial epithelium and a fibrotic lesion, respectively. Scale bars, $50 \mu \mathrm{m}$. (D) Fourteen days after bleomycin administration, the lungs were excised and collagen contents in the lungs were measured using the Sircol Collagen Assay kit. Data are shown as the mean \pm SEM for 12 mice per group. ${ }^{* *} p<0.01$ vs NS-siRNA treatment. (E) The lungs were harvested at 14 days after bleomycin administration and stained with H\&E and Masson trichrome. Scale bars, $100 \mu \mathrm{m}$. 
A

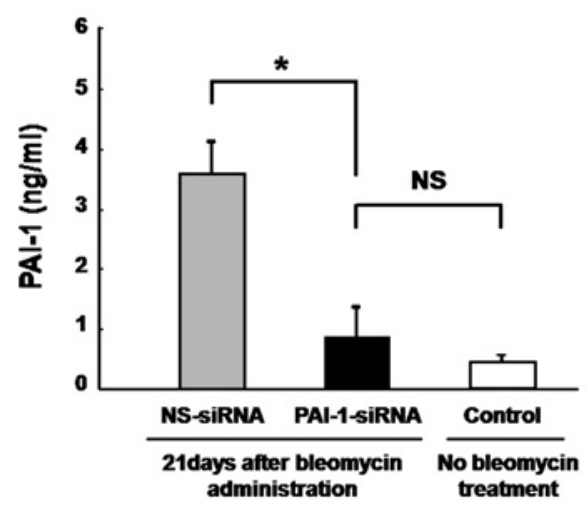

B

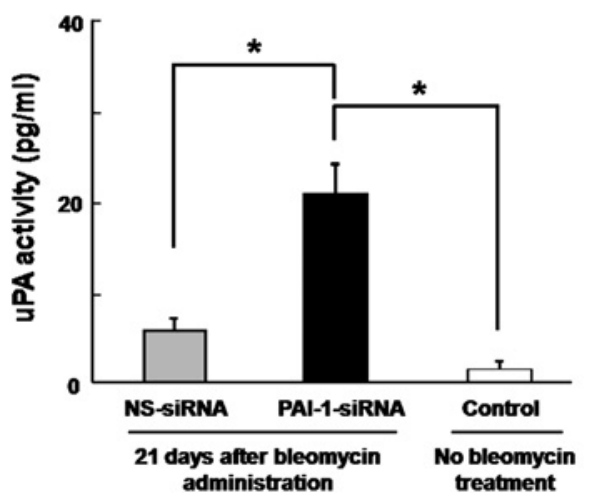

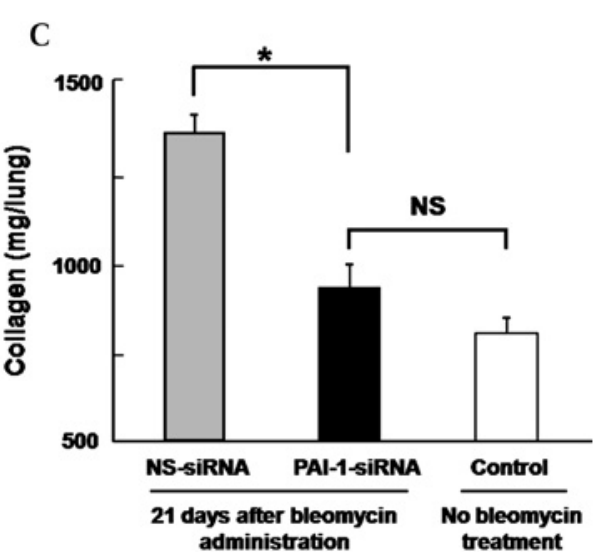

Figure 3 Effect of the repeated administration of plasminogen activator inhibitor-1 small interfering RNA (PAI-1-siRNA) started in the fibrotic phase of bleomycin-induced lung injury on the PAl-1 level, urokinase-type plasminogen activator (uPA) activity and collagen accumulation in the lungs. PAI-1siRNA or non-specific siRNA (NS-siRNA) was intranasally instilled into the mice 8, 11, 14, 17 and 20 days after bleomycin administration $(1.5 \mathrm{mg} / \mathrm{kg}$ body weight). (A) Twenty-one days after bleomycin administration, bronchoalveolar lavage (BAL) fluid was collected and the PAl-1 level in the supernatant was measured using an ELISA kit for murine PAl-1. Data are shown as the mean \pm SEM for eight mice per group. ${ }^{*} p<0.05$ vs NS-siRNA treatment. (B) Twenty-one days after bleomycin administration, BAL fluid was collected and the uPA activity in the supernatant was measured using a murine uPA activity assay kit. Data are shown as the mean \pm SEM for eight mice per group. ${ }^{*} \mathrm{p}<0.05$ vs PAl-1-siRNA treatment. (C) Twenty-one days after bleomycin administration, the lungs were excised and the collagen contents in the lungs were measured using the Sircol Collagen Assay kit. The data are presented as the mean \pm SEM for eight mice per group. ${ }^{*} \mathrm{p}<0.05$ vs NS-siRNA treatment.

\section{EMT but not apoptosis induced by TGF $\beta$ was inhibited in a mouse lung epithelial cell line transfected with PAI-1-siRNA}

LA-4 cells transfected with PAI-1-siRNA or NS-siRNA were cultured in the presence of TGF $\beta$ to assess whether the EMT or apoptotic change is affected by the introduction of PAI-1-siRNA into lung epithelial cells. As shown in figure 4C, LA-4 cells transfected with NS-siRNA morphologically changed to spindleshaped fibroblast-like cells (figure 4C); however, LA-4 cells transfected with PAI-1-siRNA retained the morphological characteristics of LA-4 cells (figure 4D). The cells were reacted with both anti- $\alpha$-SMA and anti-E-cadherin antibodies to determine whether the cells are of mesenchymal or epithelial character. As shown in figure 4G,H, LA-4 cells transfected with PAI-1-siRNA demonstrated a lower number of $\alpha$-SMA-positive cells and a higher number of E-cadherin-positive cells in comparison with those LA-4 cells transfected with NS-siRNA. When the percentages of $\boldsymbol{\alpha}$-SMA-positive cells were calculated, these numbers were significantly lower in LA-4 cells transfected with PAI-1-siRNA than in LA-4 cells transfected with NS-siRNA (figure 4M). A TUNEL assay showed no difference between LA-4 cells transfected with PAI-1-siRNA and NS-siRNA (figure 4J, K, N).

\section{DISCUSSION}

The present study demonstrated that PAI-1 is dominantly expressed in the columnar epithelial cells and hyperplastic type 2 pneumocytes lining the honeycomb lesions in the lungs of patients with IPF, and PAI-1-siRNA could distribute into the corresponding area in murine lungs with bleomycin-induced pulmonary fibrosis following intranasal administration. In addition, a single intranasal administration of PAI-1-siRNA could reduce the PAI-1 level in BAL fluid from mice with bleomycin-induced lung injury. Furthermore, the repeated administration of PAI-1-siRNA prevented the development of pulmonary fibrosis and improved the survival rate in mice with bleomycin-induced lung injury. Interestingly, the repeated administration of PAI-1-siRNA started at the beginning of the fibrotic phase of bleomycin-induced lung injury was also effective in limiting the accumulation of collagen in the lungs. In addition, the transfection of a lung epithelial cell line with PAI1-siRNA was found to inhibit EMT induced by TGF $\beta$.

The most important finding in the present study is that lungspecific suppression of PAI- 1 resulted in the prevention of pulmonary fibrosis and the improvement of survival in mice with bleomycin-induced lung injury. There is a growing body of evidence demonstrating that PAI- 1 is involved in fibrotic disorders in various organs, including the lung, kidney and liver. ${ }^{9}{ }^{14-16}$ In particular, bleomycin-induced pulmonary fibrosis is increased in mice overexpressing PAI-1 and decreased in PAI-1-knockout mice, indicating that PAI-1 is a key molecule regulating the development of pulmonary fibrosis. The results of the current study are compatible with these observations and suggest that targeting PAI-1 is a promising therapeutic strategy for pulmonary fibrosis. Indeed, a recent study that investigated the effect of a PAI-1-specific inhibitor on bleomycin-induced pulmonary fibrosis demonstrated a favourable result regarding this concept. ${ }^{17}$ Furthermore, suppressing PAI-1 specifically in the lung is a more favourable therapeutic approach because it will avoid systemic side effects that may be caused by oral administration of a PAI-1 inhibitor.

Controversy remains regarding whether the therapeutic intervention on a rodent model of pulmonary fibrosis generated by bleomycin application is anti-inflammatory or antifibrotic. The present study observed no differences in the profile of inflammatory cells recovered in BAL fluid 7 days after the intratracheal administration of bleomycin between the mice that received two courses of intranasal instillation of PAI-1siRNA and NS-siRNA. This result suggests that the suppression of PAI-1 in the bleomycin-injured lung was not an anti-inflammatory effect, and is compatible with the result of a previous study demonstrating that the extent of inflammatory injury induced by bleomycin did not differ between PAI-1-deficient mice and wild-type mice. ${ }^{14}$ Furthermore, the repeated administration of PAI-1-siRNA started during the peak inflammatory phase of bleomycin-induced lung injury was found to successfully limit the accumulation of collagen in the lung. A previous study proposed that the 'switch' between inflammation and fibrosis occurs around day 9 after intratracheal administration of 

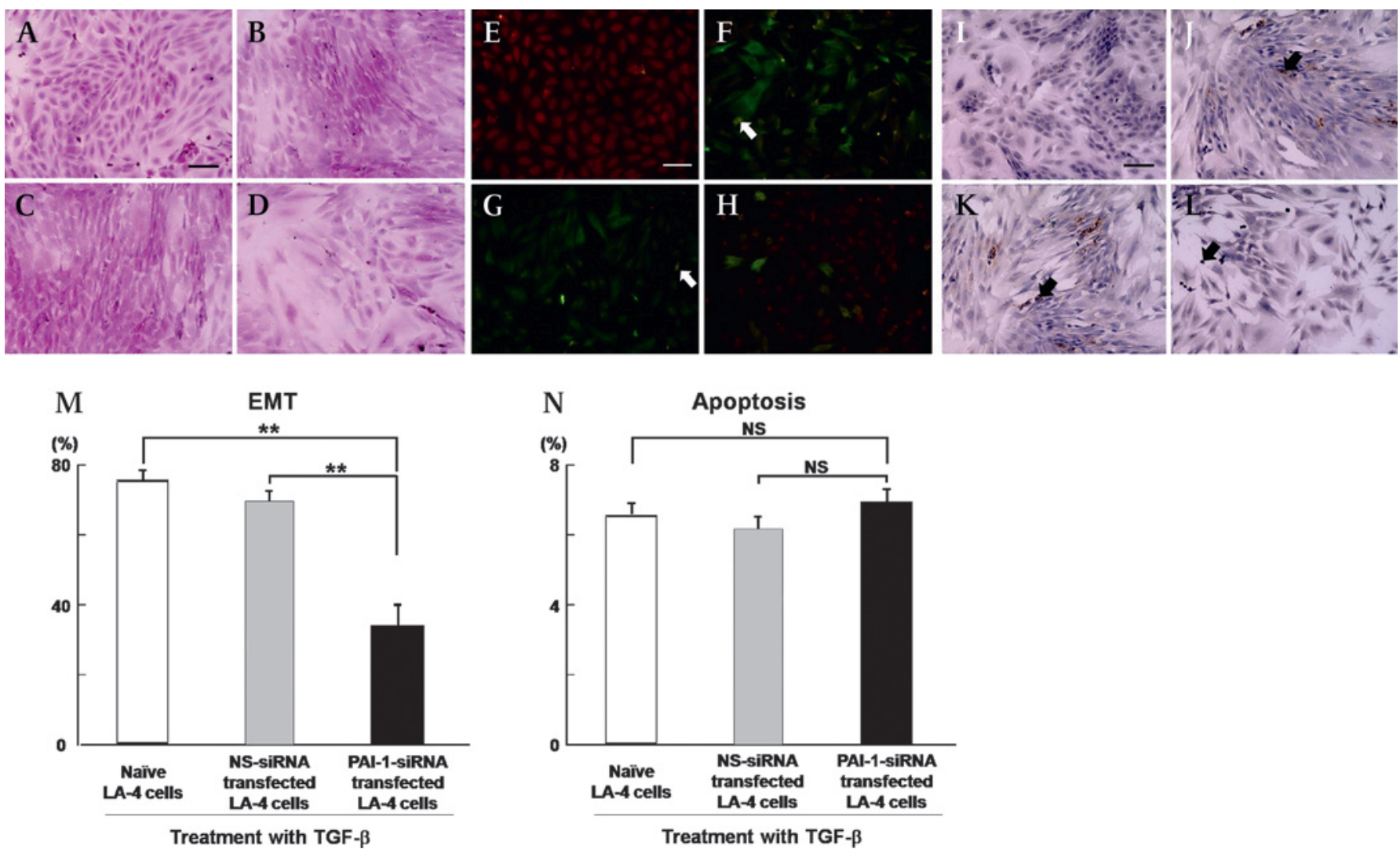

Figure 4 Assessment of epithelial to mesenchymal transition (EMT) and apoptosis induced by transforming growth factor $\beta$ (TGF $\beta$ ) in a mouse lung epithelial cell line, LA-4. (A-D) H\&E staining of LA-4 cells. (E-H) Fluorescent staining for E-cadherin (red, Alexa Fluor 594) and $\alpha$-smooth muscle actin (SMA) (green, Alexa Fluor 488) in LA-4 cells. The arrows indicate the cells containing both E-cadherin and $\alpha$-SMA (yellow). (I-L) TUNEL (terminal deoxynucleotidyl transferase-mediated dUTP nick end labelling) staining of LA-4 cells. The arrows indicate the representative cells positive for TUNEL staining. Scale bars, $100 \mu \mathrm{m}$. (A, E, I) Naïve LA-4 cells. (B, F, J) LA-4 cells treated with TGF $\beta$. (C, G, K) LA-4 cells treated with TGF $\beta$ following transfection with non-specific small interfering RNA (NS-siRNA). (D, H, L) LA-4 cells treated with TGF $\beta$ following transfection with plasminogen activator inhibitor-1 siRNA (PAI-1-siRNA). (M) Quantification of $\alpha$-SMA-positive cells in LA-4 cells treated with TGF $\beta$. The treatment with TGF $\beta$ developed significantly fewer $\alpha$-SMA-positive cells in LA-4 cells transfected with PAl-1-siRNA in comparison with naïve LA-4 cells and LA-4 cells transfected with NS-siRNA. ${ }^{* *} \mathrm{p}<0.01$ vs PAl-1-siRNA. (N) Quantification of TUNEL-positive cells in LA-4 cells treated with TGF $\beta$. The treatment with TGF $\beta$ did not cause a significant difference in the number of TUNEL-positive cells among naïve LA-4 cells, LA-4 cells transfected with NS-siRNA and LA-4 cells transfected with PAI-1-siRNA.

bleomycin, ${ }^{18}$ and a recent review on animal models of bleomycin-induced pulmonary fibrosis noted that the effect of a therapeutic intervention started $>7$ days after the last bleomycin application can be considered to be antifibrotic. ${ }^{19}$ Based on these observations, the suppression of PAI-1 by the intrapulmonary administration of PAI-1-siRNA is thought to exert an antifibrotic effect, thus resulting in the successful limitation of collagen accumulation in a mouse model of bleomycininduced pulmonary fibrosis.

Previous studies have shown the intranasal instillation of siRNA to have a significant lung-specific suppressing effect on the expression of the studied genes; however, the localisation of siRNA in the fibrotic lungs following intranasal administration remained unknown. ${ }^{10} 11$ The present study demonstrated that intranasally instilled rhodamine-labelled PAI-1-siRNA could be incorporated into the areas of the ciliated bronchial epithelium and epithelial cells lining the dense fibrotic lesions in mice lungs with bleomycin-induced pulmonary fibrosis. This result suggests that the direct administration of siRNA into the airway without using viral vectors or transfection agents is plausible for in vivo delivery of siRNA into the lung, and the gene products overexpressed in such areas of fibrotic lungs would be good targets for the suppression by siRNA. In the present study, an immuno- histochemical analysis of the lungs of patients with IPF demonstrated that PAI-1 is dominantly expressed in the areas of columnar epithelial cells and hyperplastic type 2 pneumocytes lining the honeycomb lung. A recent study that investigated PAI-1 expression in murine lungs with bleomycin-induced pulmonary fibrosis reported significantly increased expression in regenerative type 2 pneumocytes as well as alveolar macrophages. $^{20} 21$ These observations strongly suggest that PAI-1 overexpressed in the fibrotic lungs could be a good target for lung-specific delivery of siRNA.

The EMT is a process where a normal epithelial cell loses its properties and gains mesenchymal traits. Several lines of evidence indicate that the EMT plays a crucial role in the development of pulmonary fibrosis. ${ }^{22-24}$ TGF $\beta$ is one of the most important factors that induce EMT and, in fact, it is used for experiments to study EMT in fibrosis. ${ }^{25-27}$ In addition, TGF $\beta$ is also a strong inducer of PAI-1, and the involvement of PAI-1 in EMT in cancer cells has already been reported. ${ }^{28}$ In the present study, EMT induced by TGF $\beta$ in a lung epithelial cell line was shown to be inhibited by the transfection with PAI-1-siRNA. Although the precise mechanism has yet to be determined, this result indicates that PAI- 1 is involved in EMT induced by TGF $\beta$ in lung epithelial cells and probably acts as a downstream 
effector of TGF $\beta$. This result also suggests that the suppression of EMT may therefore be one of mechanisms underlying the attenuation of bleomycin-induced pulmonary fibrosis by the intrapulmonary administration of PAI-1-siRNA.

In conclusion, a single intranasal administration of PAI-1siRNA, in the absence of transfection agents, resulted in the diffuse incorporation of PAI-1-siRNA into the lining cells of the dense fibrotic area in the lung with bleomycin-induced lung injury and successfully reduced the PAI-1 level in BAL fluid. Furthermore, the repeated administration of PAI-1-siRNA started at the beginning of either the inflammatory or the fibrotic phase was effective in limiting the accumulation of collagen in a mouse model of bleomycin-induced pulmonary fibrosis. These results strongly suggest that the repeated administration of siRNA targeting PAI-1 in the lung can be a therapeutic strategy to attenuate the development and progression of pulmonary fibrosis. In addition, the EMT induced by TGF $\beta$ in a mouse epithelial cell line was inhibited by transfection with PAI-1-siRNA. The possibility that the suppression of EMT is involved in the attenuated pulmonary fibrosis by the intrapulmonary administration of PAI-1-siRNA was also demonstrated.

Funding This work was supported by a grant to the Diffuse Lung Diseases Research Group from the Ministry of Health, Labour and Welfare, Japan, and a grant from Okamoto Satoshi Memorial Fund for Pulmonary Fibrosis Research.

Competing interest None.

Provenance and peer review Not commissioned; externally peer reviewed.

\section{REFERENCES}

1. Katzenstein AL. Pathogenesis of "fibrosis" in interstitial pneumonia: an electron microscopic study. Hum Pathol 1985;16:1015-24.

2. Kuhn C 3rd, Boldt J, King TE Jr, et al. An immunohistochemical study of architectural remodeling and connective tissue synthesis in pulmonary fibrosis. Am Rev Respir Dis 1989;140:1693-703.

3. Marshall BC, Sageser DS, Rao NV, et al. Alveolar epithelial cell plasminogen activator. Characterization and regulation. J Biol Chem 1990;265:8198-204.

4. Gross TJ, Simon RH, Kelly CJ, et al. Rat alveolar epithelial cells concomitantly express plasminogen activator inhibitor-1 and urokinase. Am J Phys 1991;260: L286-95.

5. Chapman HA Jr, Stone OL, Vavrin Z. Degradation of fibrin and elastin by intact human alveolar macrophages in vitro. Characterization of a plasminogen activator and its role in matrix degradation. J Clin Invest 1984;73:806-15.

6. Idell S, James KK, Levin EG, et al. Local abnormalities in coagulation and fibrinolytic pathways predispose to alveolar fibrin deposition in the adult respiratory distress syndrome. J Clin Invest 1989;84:695-705.

7. Bertozzi P, Astedt B, Zenzius L, et al. Depressed bronchoalveolar urokinase activity in patients with adult respiratory distress syndrome. N Engl J Med 1990;322:890-7.
8. Chapman HA, Allen CL, Stone OL. Abnormalities in pathways of alveolar fibrin turnover among patients with interstitial lung disease. Am Rev Respir Dis 1986;133:437-43.

9. Eitzman DT, McCoy RD, Zheng $X$, et al. Bleomycin-induced pulmonary fibrosis in transgenic mice that either lack or overexpress the murine plasminogen activator inhibitor-1 gene. J Clin Invest 1996;97:232-7.

10. Zhang $\mathbf{X}$, Shan $\mathbf{P}$, Jiang $\mathrm{D}$, et al. Small interfering RNA targeting heme oxygenase-1 enhances ischemia-reperfusion-induced lung apoptosis. J Biol Chem 2004:279:10677-84.

11. Massaro D, Massaro GD, Clerch LB. Noninvasive delivery of small inhibitory RNA and other reagents to pulmonary alveoli in mice. Am J Physiol Lung Cell Mol Physio 2004; 287:1066-70.

12. Hattori $\mathbf{N}$, Mizuno $\mathbf{S}$, Yoshida $\mathbf{Y}$, et al. The plasminogen activation system reduces fibrosis in the lung by a hepatocyte growth factor-dependent mechanism. Am J Pathol 2004:164:1091-8.

13. Chung MP, Monick MM, Hamzeh NY, et al. Role of repeated lung injury and genetic background in bleomycin-induced fibrosis. Am J Respir Cell Mol Biol 2003;29:375-80

14. Hattori N, Degen JL, Sisson TH, et al. Bleomycin-induced pulmonary fibrosis in fibrinogen- null mice. J Clin Invest 2000;106:1341-50.

15. Gong $\mathbf{R}$, Rifai A, Tolbert EM, et al. Hepatocyte growth factor modulates matrix metalloproteinases and plasminogen activator/plasmin proteolytic pathways in progressive renal interstitial fibrosis. J Am Soc Nephrol 2003;14:3047-60.

16. Zhang LP, Takahara T, Yata Y, et al. Increased expression of plasminogen activato and plasminogen activator inhibitor during liver fibrogenesis of rats: role of stellate cells. J Hepatol 1999:31:703-11.

17. Izuhara $\mathbf{Y}$, Takahashi S, Nangaku M, et al. Inhibition of plasminogen activator inhibitor-1: its mechanism and effectiveness on coagulation and fibrosis. Arterioscler Thromb Vasc Biol 2008;28:672-7.

18. Chaudhary NI, Schnapp A, Park JE. Pharmacologic differentiation of inflammation and fibrosis in the rat bleomycin model. Am J Respir Crit Care Med 2006;173:769-76.

19. Moeller A, Ask K, Warburton D, et al. The bleomycin animal model: a useful tool to investigate treatment options for idiopathic pulmonary fibrosis? Int J Biochem Cell Biol 2008;40:362-82

20. Wygrecka M, Markart $\mathrm{P}$, Ruppert $\mathrm{C}$, et al. Cellular origin of pro-coagulant and (anti)-fibrinolytic factors in bleomycin-injured lungs. Eur Respir J 2007; 29:1105-14

21. Nishiuma T, Sisson TH, Subbotina N, et al. Localization of plasminogen activator activity within normal and injured lungs by in situ zymography. Am J Respir Cell $\mathrm{Mol}$ Biol 2004;31:552-8.

22. Kim KK, Kugler MC, Wolters PJ, et al. Alveolar epithelial cell mesenchymal transition develops in vivo during pulmonary fibrosis and is regulated by the extracellular matrix. Proc Natl Acad Sci USA 2006;103:13180-5.

23. Willis BC, Liebler JM, Luby-Phelps K, et al. Induction of epithelial-mesenchyma transition in alveolar epithelial cells by transforming growth factor-beta1: potential role in idiopathic pulmonary fibrosis. Am J Pathol 2005;166:1321-32.

24. Yao HW, Xie OM, Chen JO, et al. TGF- $\beta 1$ induces alveolar epithelial to mesenchymal transition in vitro. Life Sci 2004;76:29-37.

25. Okada H, Danoff TM, Kalluri R, et al. The early role of FSP1 in epithelialmesenchymal transformation. Am J Physiol 1997;273:563-74.

26. Miettinen PJ, Ebner R, Lopez AR, et al. TGF- $\beta$ induced transdifferentiation of mammary epithelial cells to mesenchymal cells: involvement of type I receptors. J Cell Biol 1994;127:2021-36.

27. Willis BC, Borok Z. TGF- $\beta$-induced EMT: mechanisms and implications for fibrotic lung disease. Am J Physiol Lung Cell Mol Physiol 2007;293:525-34.

28. Yoshino I, Kometani T, Shoji F, et al. Induction of epithelial-mesenchymal transition related genes by benzo[a]pyrene in lung cancer cells. Cancer 2007;110:369-74. 